\title{
TRAINIEEE: METODOLOGIA DE PROSPECÇÃO, ENGAJAMENTO E CAPACITAÇÃO DO RAMO ESTUDANTIL IEEE UFCG
}

Lara Rocha Sobral-lara.sobral@ee.ufcg.edu.br

Universidade Federal de Campina Grande

R. Aprígio Veloso, 882, Bairro Universitário

58429-900 - Campina Grande - Paraíba

Larissa da Silva Lima-larissa.lima@ee.ufcg.edu.br

Universidade Federal de Campina Grande

R. Aprígio Veloso, 882, Bairro Universitário

58429-900 - Campina Grande - Paraíba

Weverton Domingos de Medeiros - wevertonmdrs@gmail.com

Universidade Federal de Campina Grande

R. Aprígio Veloso, 882, Bairro Universitário

58429-900 - Campina Grande - Paraíba

Márcia Lais da Silva Pontes - marcia.pontes@ee.ufcg.edu.br

Universidade Federal de Campina Grande

R. Aprígio Veloso, 882, Bairro Universitário

58429-900 - Campina Grande - Paraíba

Hítalo Ricardo da Silva Lima - hitalo.lima@ee.ufcg.edu.br

Universidade Federal de Campina Grande

R. Aprígio Veloso, 882, Bairro Universitário

58429-900 - Campina Grande - Paraíba

Resumo: Este trabalho tem por objetivo apresentar uma metodologia de desenvolvimento pessoal que foi elaborada pelo Ramo Estudantil IEEE UFCG (Universidade Federal de Campina Grande) para capacitação e engajamento dos estudantes que buscam ingressar no grupo. A essa metodologia foi dado o nome de TrainIEEE e foi estruturado como um processo imersivo e qualitativo, no qual primeiramente foi feito uma análise sistemática do perfil dos inscritos, permitindo identificar suas aptidões, para que o Ramo Estudantil IEEE UFCG soubesse quais atividades e capacitações atribuir a eles. A atividade foi dividida em quatro etapas, tendo duração de cerca de dois meses, e também trabalhou as relações interpessoais do inscrito, propiciando um ambiente saudável e produtivo. A maioria dos que concluíram o processo mostraram-se bastante satisfeitos com o TrainIEEE, mostrando a capacidade do grupo de impulsionar seus voluntários, corroborando com a sua formação profissional.

Palavras-chave: Programa Trainee. Desenvolvimento pessoal. Engajamento. IEEE. 


\section{INTRODUÇÃO}

No mundo atual, as pessoas estão cada vez mais desgastadas fisicamente e psicologicamente em virtude de diversos fatores, um deles é estarem fadados a trabalhar em funções ou ambientes aos quais não se identificam e, portanto, acabam por se sentirem insatisfeitos com o trabalho que realizam. Como forma de amenizar essa situação, existem algumas soluções implementadas por empresas, uma delas é a proposta de fazer um Programa Trainee que, para Ribeiro et al (2016), é uma maneira de treinar e selecionar o candidato, à medida que o alinha aos objetivos da organização. No Programa Trainee, o candidato a determinada vaga passa um período exercendo funções relacionadas ao cargo que almeja ocupar e, com isso, é possível verificar se o mesmo se identifica e exerce bem a função, sendo positivo tanto para o funcionário que se sairá bem no que lhe for proposto, quanto à empresa que obterá bons resultados do serviço.

No cenário acadêmico, muitas vezes os estudantes são colocados em situações parecidas, pois quando não se identificam com o que estão estudando, tendem a sentir menos interesse e, consequentemente, perdem o estímulo de participar de atividades relacionadas e até mesmo chegam a trocar de curso. De acordo com Bardagi et al. (2006), no período universitário, a satisfação do estudante também pode ser relacionada a um sentimento de identificação à área de formação, no que diz respeito ao bem-estar e comprometimento. Levando em consideração que o ambiente acadêmico não se resume apenas a cursar disciplinas, mas também envolve projetos e grupos que os alunos costumam participar à exemplo de Centros Acadêmicos, Grupos de Pesquisa e Ramos Estudantis, como o IEEE, também é preciso que as pessoas se identifiquem com as tarefas que estão participando e desempenhando, porque isso implica no bom êxito do trabalho e satisfação dos participantes.

Dentre os grupos supracitados, é importante destacar que o IEEE tem alcance internacional, porque, de acordo com o próprio site do IEEE, é a maior organização profissional técnica do mundo, dedicada ao avanço da tecnologia em benefício da humanidade. Essa organização é representada por diversos Ramos Estudantis no mundo inteiro e um desses Ramos foi fundado na Universidade Federal de Campina Grande (UFCG), onde tem desempenhado importante papel na formação dos seus membros e voluntários, por meio de atividades de cunho tecnológico que atendem ao público interno e externo à comunidade acadêmica.

Dentro do IEEE, existem subgrupos chamados Capítulos Estudantis e Grupos de Afinidade. O Ramo Estudantil IEEE UFCG é composto por cinco Capítulos Estudantis, que visam aprofundamento técnico em áreas tecnológicas específicas, e um Grupo de Afinidade, Women in Engineering (WIE), que tem por objetivo estimular a participação de mulheres na área de ciências exatas.

Sabendo que o mercado de trabalho é um ambiente cada vez mais competitivo e, segundo Machado (2011), as competências dos profissionais convergem em uma gestão diferenciada e competitiva, pois agrega valor individual e corporativo, é necessário que o estudante vá além da compreensão da teoria e desenvolva novas aptidões como logística, liderança, criatividade, que serão diferenciais a seu favor ao pleitear vagas em uma empresa. Assim, o trabalho voluntário em organizações como o IEEE permite que o estudante possa complementar sua formação profissional.

Mas, como toda organização, o engajamento dos participantes é fundamental para o sucesso. Pensando nisso, o Ramo Estudantil IEEE UFCG estudou e analisou a situação do grupo a fim de compreender o perfil dos participantes e, assim, encontrar a razão da falta de engajamento de alguns. 
A partir da análise, notou-se que a afinidade com a atividade desempenhada é um ponto crucial no estímulo que promove a retenção dos voluntários no grupo. Com essa constatação, o desafio seria organizar a entrada de novos voluntários, de modo que fosse possível reconhecer nos novatos suas habilidades, para, assim, associá-los aos subgrupos e funções de acordo com seus respectivos perfis.

Tendo como base essa necessidade, a diretoria do Ramo Estudantil IEEE UFCG idealizou um modelo de trainee para ser aplicado no início do semestre letivo, com o intuito de realizar dinâmicas de grupo entre os inscritos, que promoviam um momento de imersão nos princípios e valores do IEEE, bem como entrevistas com perguntas bem elaboradas e diretivas que permitissem reconhecer nos entrevistados suas habilidades e afinidades com as áreas em questão.

A esse modelo idealizado, foi dado o nome de "TrainIEEE", como referência aos processos seletivos de empresas e ao IEEE. Para explicar o trabalho desenvolvido pelo Ramo Estudantil IEEE UFCG a partir do TrainIEEE e seus respectivos resultados obtidos na primeira edição da atividade, o artigo está organizado da seguinte forma: a Seção 2 apresenta a metodologia da atividade; a Seção 3 apresenta os principais resultados obtidos com a realização da atividade no ano de 2019 e as discussões; na Seção 4 são apresentadas as considerações finais.

\section{METODOLOGIA}

O TrainIEEE é uma atividade que busca prospectar, orientar e engajar novos voluntários para o Ramo Estudantil IEEE UFCG. Por meio de um processo imersivo que tinha por objetivo identificar as aptidões dos interessados e treiná-los para exercerem funções com as quais se identificassem, essa atividade foi estruturada em quatro etapas e teve duração de cerca de cinquenta dias.

Apesar da contribuição na formação curricular, muitas vezes o estudante se engajava em atividades que com as quais não tinham afinidade, o que acabava por desestimular o novo voluntário. Desse modo, o Ramo Estudantil IEEE UFCG estruturou um processo qualitativo: o TrainIEEE, que busca identificar as aptidões daqueles que buscavam ingressar no grupo, para depois lhes atribuir atividades que correspondem as suas competências. Sob essa ótica existem metodologias com o mesmo princípio, a exemplo da metodologia DISC (Dominance Influence Steadiness Conscientiousness), onde, segundo Marston (1989), é possível avaliar quatro principais fatores do comportamento humano: Dominância, Influência, Estabilidade e Conformidade.

Tendo em vista que o IEEE é uma organização com viés tecnológico e filantrópico, a atividade buscou identificar quatro diferentes perfis de voluntários:

1. Perfil humanitário: pessoas fortemente empáticas a problemas sociais;

2. Perfil gestor: pessoas com aptidões a liderança;

3. Perfil criativo: pessoas com facilidade para criar, inventar e imaginar;

4. Perfil operacional: pessoas com aptidões a logística.

Desse modo, as duas primeiras etapas da atividade consistiram em identificar os perfis supracitados, e as outras duas trabalharam o seu engajamento no grupo e o aperfeiçoamento das suas aptidões naturais.

A primeira etapa do processo ocorreu online por meio de um formulário de inscrição, que permitiu traçar os perfis sociais dos inscritos. Nele, havia dez questões de múltipla escolha, nas quais cada uma das respostas se relacionava com uma das áreas administrativas do Ramo Estudantil IEEE UFCG, possuindo, consequentemente, atribuições específicas. Vale ressaltar 
que o participante poderia se enquadrar em mais de um perfil e, consequentemente, poderia ser encaixado em mais de uma tarefa.

A segunda etapa ocorreu presencialmente durante um dia. Nela, inicialmente, buscou-se explicar no que consistia o trabalho do IEEE e mostrar a importância do voluntariado na formação humana. Após esse momento inicial foram realizadas duas dinâmicas que permitiram atestar ou contestar os resultados obtidos no formulário, uma vez que o mesmo é bastante limitado para traçar um perfil. Dessa maneira, no primeiro exercício os participantes tiveram que confeccionar seu próprio cartaz, permitindo que, além de ser possível identificá-los pelo nome, fosse possível analisar as suas habilidades criativas.

No segundo exercício, os inscritos foram divididos em grupos de até cinco pessoas e tiveram quinze minutos para criar um cartaz sobre um dos 17 Objetivos do Desenvolvimento Sustentável (ODS) da agenda mundial das Nações Unidas para 2030 e, em seguida, tinham que fazer um Pitch de apresentação do trabalho. Nessa dinâmica, pessoas com diferentes perfis foram colocadas no mesmo grupo, permitindo que fosse possível analisar sua habilidade de trabalhar em equipe. Além disso, o exercício permitia observar o engajamento social, planejamento estratégico, liderança, criatividade, organização de tempo e logística de execução dos participantes. Na Figura 1, pode-se ver as equipes e seus respectivos pôsteres.

Figura 1 - Dinâmica dos pôsteres sobre as 17 ODS.

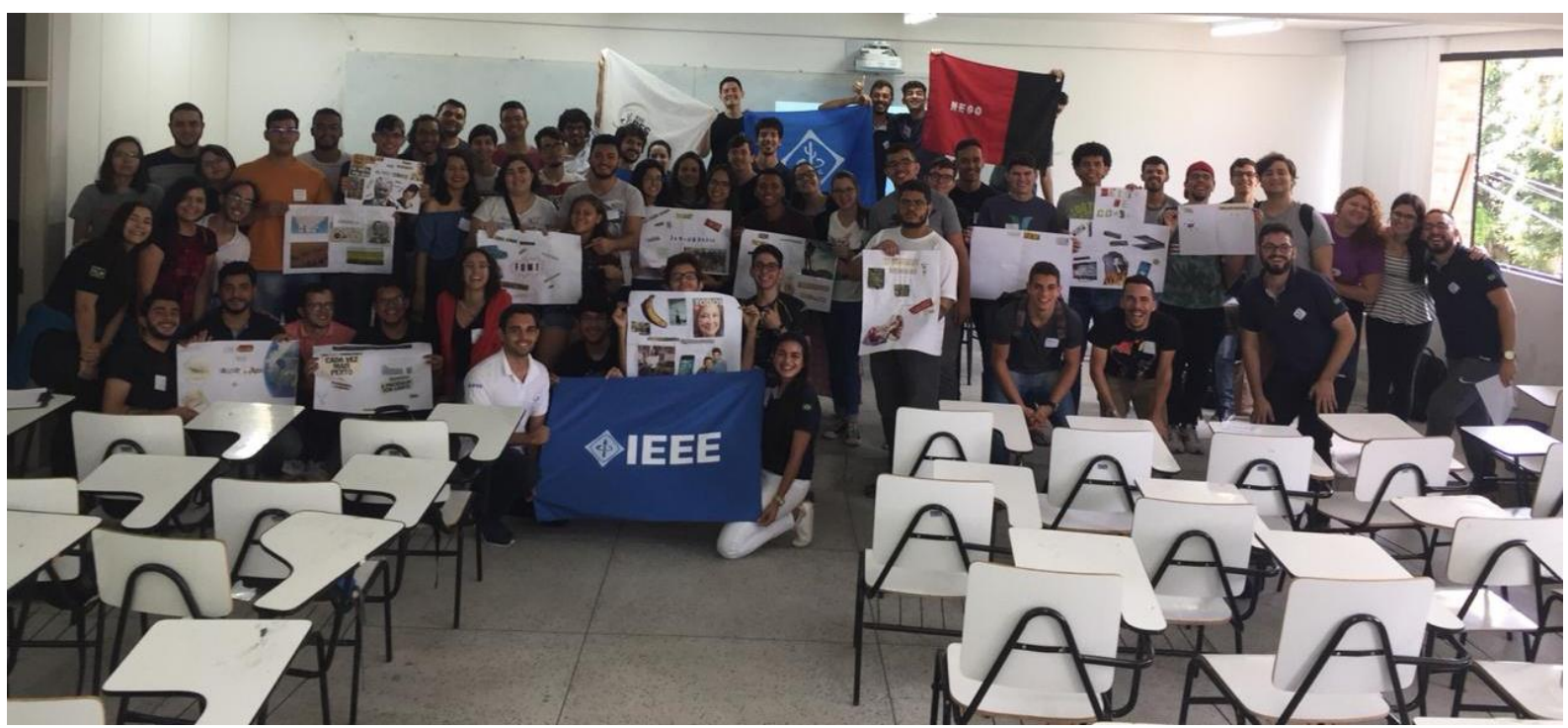

Fonte:Autor

Durante as dinâmicas, cada uma das equipes tinha dois monitores, que os acompanhavam e tiravam suas dúvidas. Ademais, os monitores possuíam uma ficha que continha as informações do perfil de cada um dos inscritos que estavam sob sua observação. Assim, eles poderiam adicionar comentários sobre as suas habilidades, complementando o resultado da primeira etapa. A Figura 2 mostra as equipes discutindo sob a supervisão de seus monitores. res. 


\section{COBENGE C.COBENGE: 2020 da ABENGE}

"Os desafios para formar hoje o engenheiro do amanhã"

Figura 2 - Avaliação dos monitores.

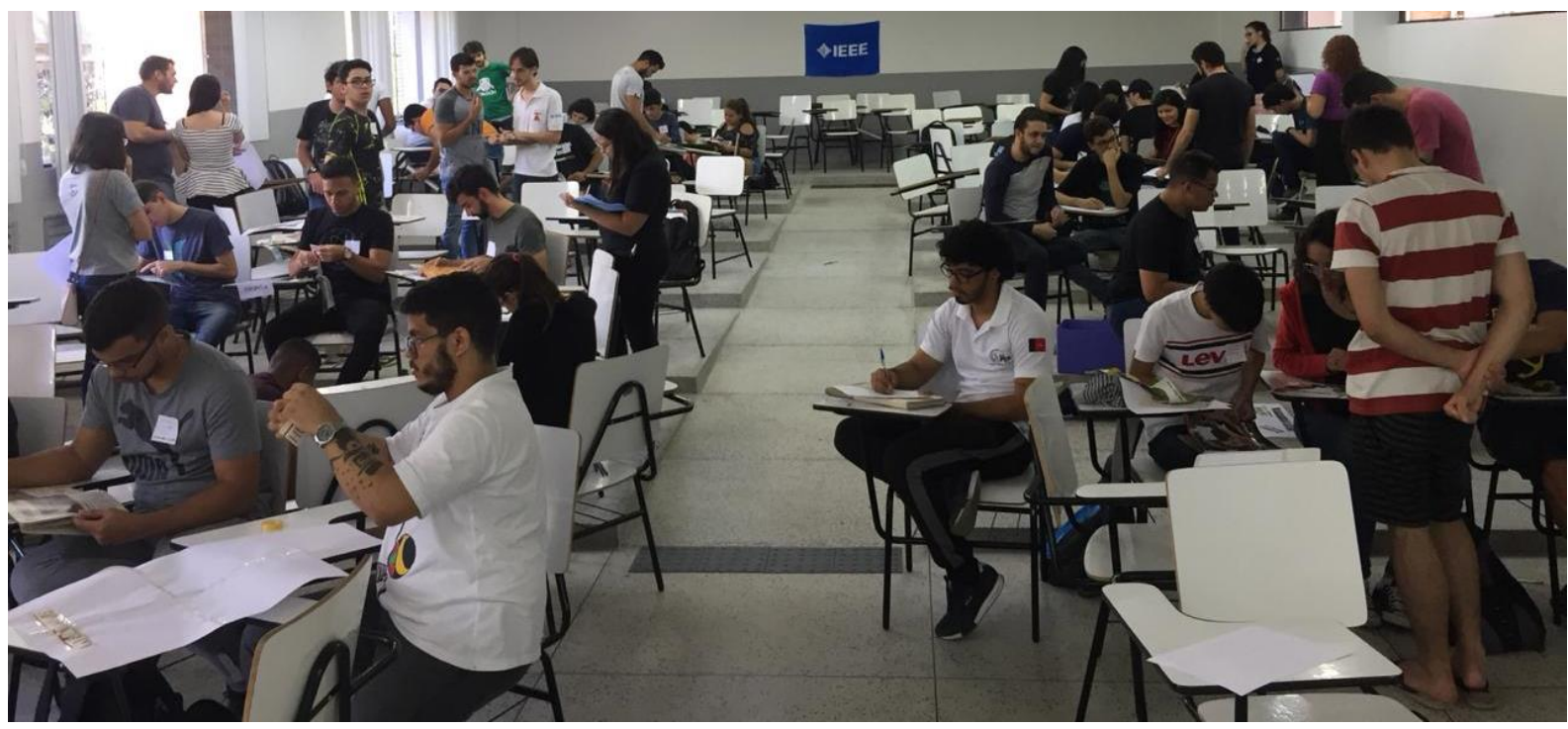

Fonte:Autor

Encerrados os exercícios, os participantes foram dirigidos a entrevistas com os responsáveis pelas diretorias do Ramo Estudantil IEEE UFCG, seus capítulos e o grupo de afinidade, permitindo que os inscritos pudessem conhecer as pessoas com as quais trabalhariam durante a etapa seguinte. Para este momento, os entrevistadores receberam as fichas com os comentários dos monitores e, aliado à conversa com os participantes, puderam formar um perfil conclusivo.

A terceira etapa foi um estágio que teve duração de vinte e cinco dias. Nela, os participantes tiveram uma experiência de imersão, para que pudessem conhecer melhor o Ramo Estudantil. Assim, com base no seu perfil, foram-lhes atribuídas atividades para que eles realizassem ao longo das semanas, fazendo com que assumissem, sob orientação, algumas responsabilidades dentro do grupo. Além disso, essa fase permitiu que eles desenvolvessem suas aptidões, por meio das capacitações, e outras habilidades importantes como trabalho em equipe e gestão de tempo. Nessa etapa, os participantes também conheceram ferramentas de gerenciamento organizacional que são utilizadas pelo Ramo Estudantil IEEE UFCG, como o Trello e o Slack.

Vale ressaltar que na terceira fase a comunicação dos inscritos também foi trabalhada, tanto internamente quanto externamente. Internamente, as diretorias fizeram grupos em redes sociais com os inscritos no TrainIEEE, fazendo com que se sentissem acolhidos e pudessem se comunicar com os voluntários, tirando dúvidas e criando laços com os membros mais antigos da equipe. Externamente, essas tarefas que os voluntários do TrainIEEE ficaram responsáveis foram abertas ao público, indo desde atividades de conversação em língua estrangeira para alunos da UFCG, até oficinas de ciências com estudantes de ensino médio de escolas públicas. Nessas tarefas, eles foram incentivados a tomarem a frente, ministrando as atividades, aprimorando assim as suas oratórias.

Por fim, a quarta etapa consistiu em um momento de celebração e recepção dos novos voluntários. Nessa fase, foi assinado o termo de voluntariado, um documento simbólico que representa o compromisso e o desejo do participante de ingressar no Ramo Estudantil IEEE UFCG. Também foi realizada uma última dinâmica onde os participantes tiveram que escrever em um papel o que o TrainIEEE havia significado para eles. Essa tarefa permitiu que o Ramo Estudantil IEEE UFCG obtivesse um feedback da atividade, a fim de compreender as 
impressões dos participantes sobre o processo. Na Figura 3 é possível ver o painel formado pelos papeis com a avaliação.

Figura 3 - Feedback da atividade.

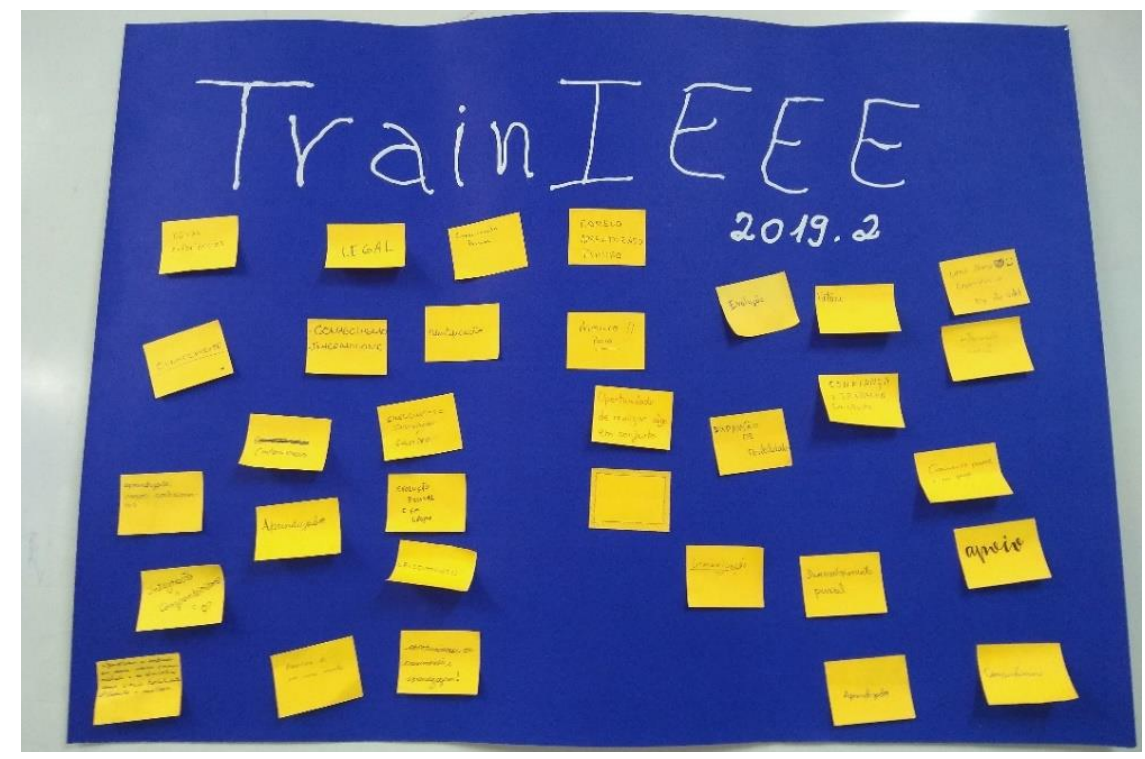

Fonte:Autor

Vale ressaltar ainda que toda a documentação desenvolvida ao longo da construção do processo foi salva em um drive de acesso aos responsáveis pelo Ramo Estudantil IEEE UFCG, de modo a permitir que a atividade pudesse ser replicada e aprimorada em edições futuras.

\section{RESULTADOS E DISCUSSÕES}

Tendo em vista que a missão do IEEE é promover o avanço tecnológico em benefício da humanidade, espera-se que ele e suas representações dentro das universidades, como o Ramo Estudantil IEEE UFCG, busquem ser agentes transformadores nos ambientes onde estão inseridos. Para que isso ocorra, a transformação deve ocorrer primeiramente dentro do grupo e dos voluntários, sendo externada posteriormente.

O TrainIEEE ocorreu durante os dias 19 de agosto e 9 de outubro, durante o semestre de 2019.2, na UFCG em um processo que favoreceu tanto o Ramo Estudantil IEEE UFCG quanto os seus participantes. Por parte do grupo responsável, permitiu um crescimento contínuo do número de voluntários ativos, tendo aumento de 50 novos integrantes, que foram capacitados e integrados com sucesso ao âmbito do grupo.

Também é válido ressaltar que, dentro do IEEE existe um sistema de associação por meio de membresia, que garante aos Ramos Estudantis visibilidade internacional, atrelada ao aumento do número de membros. Isso possibilita que o grupo tenha maior suporte organizacional por parte do IEEE, para realizar atividades. À vista disso, o TrainIEEE contribuiu para que o Ramo Estudantil IEEE UFCG adquirisse mais 12 novas membresias, totalizando 112 ao final de 2019, sendo 99 de membresias estudantis.

Sob a ótica dos novos voluntários, esses passaram por um processo que lhes ajudou a identificar suas competências e habilidades, podendo aperfeiçoar seus talentos e desenvolver novas aptidões extremamente necessárias no mercado de trabalho, como oratória, trabalho em equipe, gestão de tempo, entre outros. Além disso, o processo permitiu o fortalecimento do 
vínculo entre grupo e os novos participantes, o que é atestado pelo fato de que desses novos voluntários, 10 assumiram cargos de responsabilidade durante a gestão de 2020 .

Com o intuito de obter uma avaliação dos voluntários que participaram do TrainIEEE e permaneceram até a fase de assinatura do termo de voluntariado, foi enviado um formulário por e-mail para cada um dos participantes, a fim de observar de maneira quantitativa e qualitativa o desempenho da atividade mediante a opinião de quem vivenciou de fato.

No total, foram 28 respostas à 8 perguntas, sendo a última delas aberta e não obrigatória, para que os voluntários se sentissem confortáveis ao compartilhar suas críticas e sugestões. Dentre algumas das perguntas realizadas, foi questionado o grau de satisfação com o TrainIEEE e $57,1 \%$ dos votantes classificaram como "Excelente", como mostra a Figura 4. Uma pessoa ainda adicionou uma opção "TOP”, uma palavra de origem inglesa cuja tradução implica em algo que seja superior, esteja acima.

Figura 4 - Grau de satisfação na atividade.

Qual seu grau de satisfação com o TrainlEEE?

28 respostas

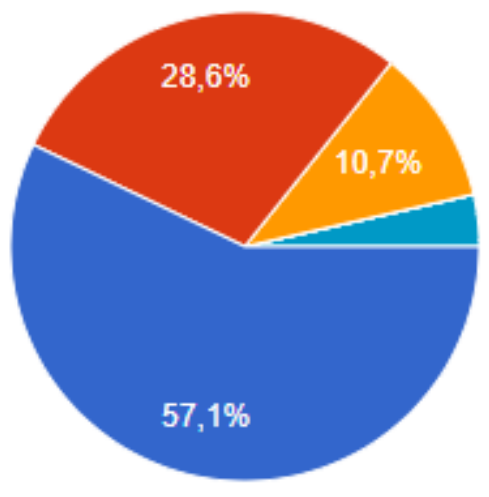

Excelente

Bom

Regular

Ruim

Péssimo

TOP

Fonte:Autor

Outra pergunta do formulário estava relacionada à contribuição da atividade para o engajamento do novato no IEEE. Para esta, 92,9\% responderam que sim, conforme a Figura 5. 
Figura 5 - Contribuição para o engajamento.

O TrainIEEE contribuiu para o seu engajamento no IEEE?

28 respostas

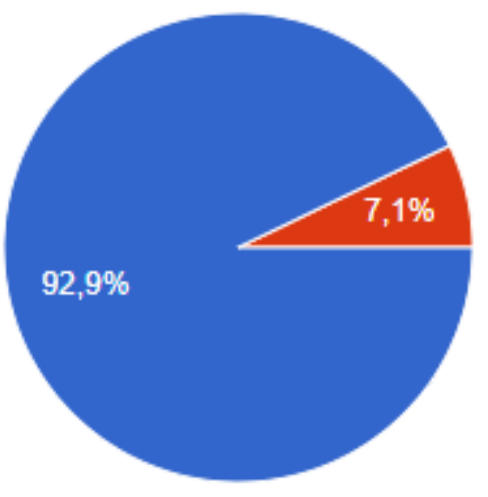

Fonte:Autor

Reforçando a preocupação do Ramo Estudantil IEEE UFCG em promover atividades que contribuam para a formação de seus membros e voluntários, um dos questionamentos foi se o TrainIEEE havia ajudado na formação do participante enquanto estudante de graduação. Tal como é ilustrado na Figura 6, dos 28 que responderam, 89,3\% confirmaram a contribuição.

Figura 6 - Contribuição para a formação estudantil.

O TrainIEEE ajudou na sua formação enquanto estudante?

28 respostas

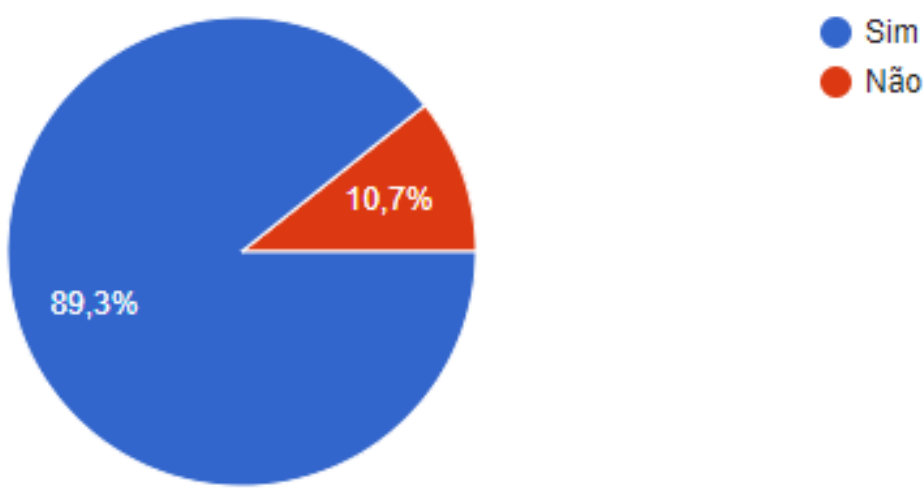

Fonte:Autor

Por fim, mas antes de solicitar as críticas e sugestões, foi perguntado se o novo voluntário recomendaria o TrainIEEE para um amigo e apenas uma pessoa respondeu que não, assim comprovado na Figura 7. 
Figura 7 - Recomendação para terceiros.

Você indicaria o TrainIEEE do Ramo Estudantil IEEE da UFCG para um amigo?

28 respostas

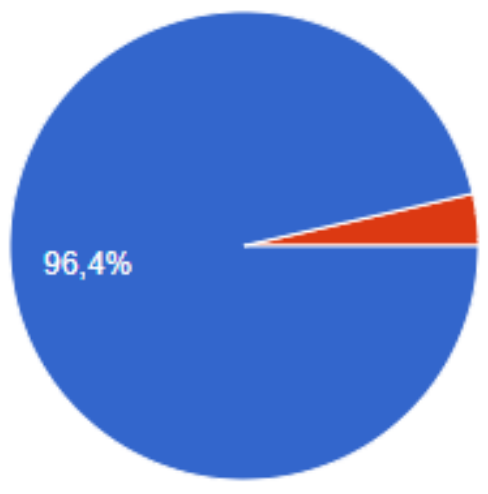

Sim

Não

Fonte:Autor

No que diz respeito às críticas e sugestões, os comentários giraram em torno de grande satisfação por ter participado da atividade, à exemplo de uma resposta em que a voluntária relata "[...] Além de uma grande troca de experiências, recebi todo o apoio e acolhimento nos momentos iniciais com os capítulos, o que me motivou e me deixou mais engajada nas atividades.".

Quanto às sugestões, foi levantada a questão de procurar aumentar o alcance da atividade, a fim de que pessoas dos mais variados cursos participassem, o que já é viabilizado pelo modelo da atividade, no entanto, esbarra nas problemáticas de muitos ainda considerarem um grupo voltado apenas à área de ciências exatas, além do distanciamento relacionado a participação e comunicação existente entre alguns cursos da universidade. Outro ponto levantado foi o de manter a dinâmica de divulgação para que todos os membros do Ramo Estudantil IEEE UFCG estejam cientes das atividades que são realizadas pelo grupo.

\section{CONSIDERAÇÕES FINAIS}

De modo geral, mesmo estando apenas em sua primeira edição, o TrainIEEE é uma atividade que trouxe resultados muito positivos tanto para os candidatos, quanto para os organizadores, tendo em vista que esses últimos tiveram a oportunidade de estar a frente de um processo que provavelmente podem vir a participar na carreira profissional, seja como candidato ou coordenador. Nesse sentido, a familiaridade com o processo certamente será um fator facilitador.

Diante disso, o Ramo Estudantil IEEE UFCG se destaca dentro da academia ao impulsionar seus voluntários, por meio de atividades que compreendem categorias técnicas e sociais, a estarem cada vez mais preparados tanto para o ambiente educacional, como para o mercado de trabalho, visto que possibilita o desenvolvimento de competências e habilidades importantes.

Analisados os pontos mencionados no questionário, torna ainda mais perceptível o crescimento do grupo e o quanto atividades como o TrainIEEE se tornam fundamentais, dado que fazer e se sentir parte de um grupo é algo muito importante no que diz respeito à vivência em sociedade. 
Assim como um projeto piloto, essa atividade pode ser ainda mais aprimorada e demonstra potencial para isso, pois tem como fundamento não apenas os resultados, mas o cuidado com os que são responsáveis por esses resultados acontecerem. Destarte, a preocupação com o engajamento e a afinidade dos envolvidos em um contexto de um grupo, propicia um ambiente mais saudável e produtivo.

\title{
Agradecimentos
}

Agradecemos a gestão do Ramo Estudantil IEEE UFCG de 2019 pela idealização e execução do TrainIEEE, bem como a contribuição de todos os voluntários que fazem parte desse grupo.

\section{REFERÊNCIAS}

BARDAGI, Marúcia et al. Escolha profissional e inserção no mercado de trabalho: percepções de estudantes formandos. Psicologia escolar e educacional, v.10, n.1, p. 69-82, 2006.

IEEE Disponível em: http://www.ieee.org/ Acesso em: 30 mai. 2020.

MACHADO, Mirian Magnus; DE CAMARGO SANTOS, Regina Maria. A gestão de competências como diferencial competitivo apresentada a acearia Frederico Missner S/A. . Revista Interdisciplinar Científica Aplicada, v.5, n.3, p. 61-88, 2011.

MARSTON, William Moultan. Emotions of normal people. Ormskirk: Thomas Lyster Ltd.,1989.

RIBEIRO, Andréia Miguens et al. Desenvolvimento de competências de trainee de empresas juniores. In: XXI Congresso Nacional de Administração, 2016, Goiânia. Anais. Goiânia, 2016.

\section{TRAINIEEE: PROSPECTING, ENGAGEMENT AND TRAINING METHODOLOGY OF THE IEEE UFCG STUDENT BRANCH}

\begin{abstract}
This work intends to present a methodology of personal development that was elaborated by the IEEE UFCG Student Branch (Federal University of Campina Grande) for training and engagement of the students who seek to join the group. This procedure was given the name TrainIEEE and was structured as an immersive and qualitative process, in which a systematic analysis of the subscriber's profile was first carried out, allowing them to identify their aptitudes, so that the IEEE UFCG Student Branch knew which activities and capabilities to assign to them. The activity was divided into four stages, lasting two months, and also worked on the interpersonal relationships of the enrollee, providing a healthy and productive environment. Most of those who completed the process were very satisfied with TrainIEEE, showing the group's ability to boost its volunteers, corroborating their professional training.
\end{abstract}

Keywords: Trainee Program, personal development, engagement, IEEE. 